\title{
Principaux conseils cliniques en matière de soins primaires pour participer à l'élimination de la tuberculose au Canada
}

\author{
Amaratunga, $\mathrm{KR}^{1,2 *}$, Alvarez, $\mathrm{GG}^{3,4}$
}

\section{Résumé}

Bien que l'incidence de la tuberculose soit faible au Canada, certaines populations, y compris les peuples autochtones nés à l'étranger ou au Canada, sont représentées de manière disproportionnée parmi les cas déclarés. Les taux d'incidence globaux de tuberculose active au Canada n'ont pas connu de baisse significative au cours de la dernière décennie et il reste du travail à faire pour atteindre les objectifs d'élimination de la maladie définis par l'Organisation mondiale de la Santé (OMS). Pour parvenir à éliminer la tuberculose au Canada, les cliniciens en soins primaires, avec l'aide des professionnels de la santé publique et des experts de la maladie, peuvent participer à l'effort en mettant l'accent sur 1) le dépistage ciblé et le traitement des infections tuberculeuses latentes et 2) le diagnostic en temps opportun de la tuberculose active et la référence de ces cas. L'article suivant met l'accent sur certains des principaux aspects des soins primaires à garder à l'esprit dans les soins quotidiens aux patients. Pour effectuer un dépistage ciblé des infections tuberculeuses latentes et les traiter, plusieurs populations clés, y compris les immigrants venant de pays très touchés par la tuberculose, les peuples autochtones, et plusieurs autres groupes à risque, sont identifiées. La réactivation des infections tuberculeuses latentes joue un rôle important dans le fardeau de la tuberculose et pourrait avoir une incidence majeure dans les efforts pour éliminer la tuberculose. Les progrès réalisés en matière de traitement des infections tuberculeuses latentes, y compris les traitements à court terme, sont également décrits. En outre, pour aider à diagnostiquer la tuberculose active en temps opportun, plusieurs facteurs de risque, y compris un certain nombre de comorbidités, qui augmentent les risques de développer la maladie, peuvent être pris en compte. En prodiguant des soins de première ligne aux patients, les fournisseurs de soins peuvent participer à l'élimination de la tuberculose au Canada s'ils gardent à l'esprit ces conseils clés.

\begin{abstract}
Affiliations
${ }^{1}$ Centre de la lutte contre les maladies transmissibles et les infections, Agence de la santé publique du Canada, Ottawa (Ontario)
\end{abstract}

2Département de médecine de I'Université d'Ottawa, division des maladies infectieuses, Ottawa (Ontario)

${ }^{3}$ Département de médecine de l'Université d'Ottawa, épidémiologie et médecine communautaire, division de pneumologie et des maladies infectieuses, Ottawa (Ontario)

${ }^{4}$ Institut de recherche de l'Hôpital d'Ottawa, Ottawa (Ontario)

*Correspondance : kanchana. amaratunga@phac-aspc.gc.ca
Citation proposée : Amaratunga KR, Alvarez GG. Principaux conseils cliniques en matière de soins primaires pour participer à l'élimination de la tuberculose au Canada. Relevé des maladies transmissibles au Canada 2016;42:72-7. https://doi.org/10.14745/ccdr.v42i03a03f

\section{Introduction}

En mai 2014, I'Assemblée mondiale de la santé a approuvé la stratégie mondiale de lutte antituberculeuse après 2015 de l'Organisation mondiale de la Santé (OMS) visant à mettre fin à la maladie d'ici 2035. L'un des objectifs ambitieux est de réduire le taux d'incidence global de la tuberculose pour qu'il soit inférieur à 100 cas pour un million de personnes. Cela nécessiterait de réduire de $95 \%$ le nombre de décès dus à la maladie, et de $90 \%$ le taux d'incidence de la tuberculose d'ici 2035, par rapport à 2015 (1). Pour les pays comme le Canada où le taux d'incidence est faible, et qui ont déjà atteint I'objectif d'une incidence inférieure à 100 cas pour un million de personnes, la stratégie mondiale a été adaptée afin de fournir un cadre de référence pour l'élimination de la tuberculose (définie comme un taux inférieur à 1 cas pour un million de personnes) d'ici 2050 (1).
Malgré le faible taux d'incidence de la maladie au Canada, il reste du travail à faire pour éliminer la tuberculose dans notre pays. D'après le Plan mondial Halte à la tuberculose 2006-2015 (OMS) (2), l'objectif du Canada était d'atteindre un taux d'incidence de 3,6 cas par an pour 100000 personnes avant 2015, un objectif réaffirmé dans le document intitulé Prévention et contrôle de la tuberculose au Canada : un cadre d'action fédéral, publié en 2014 (3). Entre 2003 et 2013, le taux d'incidence global signalé de tuberculose au Canada est passé de 5,2 cas à 4,7 cas pour 100000 personnes (4). Bien que l'incidence de la tuberculose active dans la population générale canadienne soit l'une des plus faibles au monde, les taux n'ont pas connu de changement significatif au cours de la dernière décennie. Les personnes autochtones nées à l'étranger et au Canada sont toujours représentées de façon disproportionnée parmi les cas déclarés. En 2013, les personnes nées à l'étranger, qui composaient environ $22 \%$ de la population canadienne, représentaient $71 \%$ des cas déclarés, et les personnes 
autochtones nées au Canada, qui constituent $4 \%$ de la population canadienne, représentaient $19 \%$ des cas déclarés (4).

Le cadre d'action de l'Organisation mondiale de la Santé cite plusieurs obstacles à l'élimination de la tuberculose dans les pays où l'incidence de la maladie est faible, notamment la baisse de l'expertise clinique et diagnostique, ainsi que la diminution de la sensibilisation à la tuberculose due à une incidence faible (1). Dans ces pays, l'un des huit domaines d'action prioritaires est le dépistage des infections tuberculeuses latentes et de la tuberculose active dans les groupes de contact et les groupes à haut risque sélectionnés, ainsi que l'administration de traitements adaptés (1).

Les cliniciens en soins primaires prodiguent des soins à des personnes de différents milieux et s'occuperont donc inévitablement des populations présentant un risque plus élevé de tuberculose et d'infection tuberculeuse. À mesure que les taux d'incidence de la tuberculose diminuent au Canada, le niveau global d'expérience des médecins avec la maladie diminuera également. Ainsi, même s'il est très important que des médecins expérimentés prennent en charge les cas de tuberculose, il est tout aussi prioritaire d'informer les médecins référants, y compris les cliniciens en soins primaires, des derniers progrès en matière de dépistage et de prise en charge de la tuberculose (5).

Les cliniciens en soins primaires, avec l'aide des professionnels de la santé publique et des experts de la tuberculose, jouent deux rôles clés dans les efforts d'élimination au Canada : effectuer un dépistage ciblé des infections tuberculeuses latentes et les traiter, ce qui évite leur réactivation et permet d'accélérer la diminution de l'incidence de la maladie, et diagnostiquer la tuberculose active en temps opportun afin de prévenir sa transmission et de contribuer au maintien de la baisse de l'incidence.

\section{Dépistage ciblé et traitement des infections tuberculeuses latentes}

D'après les récentes données présentées devant l'Advisory Council for the Elimination of Tuberculosis (ACET) des Centers for Disease Control and Prevention en décembre 2015, le nombre de cas de tuberculose dus à une réactivation des infections tuberculeuses latentes pourrait être plus élevé qu'on ne le pensait auparavant (6). Les membres de l'Advisory Council ont établi que le ciblage des infections tuberculeuses latentes constituerait une action dont les répercussions seraient plus importantes pour atteindre les objectifs d'élimination de la tuberculose. Toutefois, il s'agit d'une lourde tâche.

Chaque année, environ 250000 immigrants et réfugiés s'installent au Canada $(7,8)$. Dans le cadre de l'examen médical réglementaire effectué sur les personnes déposant une demande de résidence permanente, et de certaines catégories de résidence temporaire, les personnes âgées de 11 ans et plus devraient passer une radiographie des poumons dans leur pays d'origine $(8,9)$. En fonction des antécédents et de l'examen physique réalisé dans le cadre de l'examen médical réglementaire, toute personne soupçonnée d'être atteinte de tuberculose doit être référée pour des examens supplémentaires dans son pays d'origine, et toute personne chez qui une tuberculose active a été diagnostiquée devrait terminer son traitement avant d'arriver au Canada (7).

La surveillance post-immigration des immigrants sélectionnés et considérés comme présentant un risque élevé de développer une tuberculose active dépend également de certains facteurs de risque identifiés lors de l'examen médical réglementaire (7). L'objectif de l'examen médical réglementaire est de s'assurer que les migrants atteints de tuberculose active sont diagnostiqués et traités, et qu'ils ne sont plus contagieux avant leur arrivée au Canada. Le but n'est pas de repérer ou de traiter les infections tuberculeuses latentes (8). Ni le test cutané à la tuberculine (TST), ni le test de libération d'interféron-gamma (TLIG) ne sont effectués systématiquement sur les nouveaux arrivants au Canada. Un dépistage de masse de l'ensemble de cette population n'est pas recommandé, car il serait impossible à mettre en œuvre sur le plan logistique et se révélerait en grande partie inefficace.

La sélection des individus pour le dépistage ciblé et le traitement des infections tuberculeuses latentes dépend de leur risque d'exposition antérieure à la tuberculose et du risque de réactivation, ainsi que de la probabilité qu'ils aient terminé le traitement de manière sûre, ce qui comprend le risque d'hépatotoxicité (10). II n'est pas toujours évident de déterminer quels patients peuvent bénéficier d'un dépistage et d'un traitement des infections tuberculeuses latentes. Le tableau 1 a été établi à partir des dernières Normes canadiennes pour la lutte antituberculeuse, 7e édition élaborées par la Société canadienne de thoracologie et l'Agence de la santé publique du Canada. Il présente brièvement sept groupes sélectionnés parmi différentes populations vulnérables que les médecins de première ligne peuvent rencontrer souvent dans leur pratique et les personnes pour lesquelles ils devraient envisager un dépistage cibler des infections tuberculeuses latentes.

Un outil en ligne gratuit a été mis à disposition par l'Université McGill (http://www.tstin3d.com/) afin d'aider à interpréter les résultats du test cutané à la tuberculine ou du test de libération d'interféron-gamma en estimant le risque de présenter une tuberculose active à l'aide des facteurs de risque présentés dans le tableau 1 (14). Les cliniciens doivent néanmoins toujours prendre en compte le risque de réactivation du patient et la capacité à effectuer un suivi continu si un dépistage et un traitement sont proposés. La probabilité de terminer le traitement de manière sûre, y compris la possibilité d'effectuer un suivi continu pour surveiller les effets indésirables potentiels tels que l'hépatotoxicité pendant le traitement, doit être prise en compte (10). Si un dépistage est proposé et que l'on soupçonne une infection tuberculeuse latente chez le patient à cause d'un test cutané à la tuberculine ou d'un test de libération d'interféron-gamma positif, il faut s'assurer d'exclure une tuberculose active avant de démarrer un traitement contre I'infection tuberculeuse latente. Pour exclure une tuberculose active, il faut généralement effectuer un dépistage des symptômes, une radiographie pulmonaire, un frottis et une culture d'expectoration. 
Tableau 1 : Recommandations de la Société canadienne de thoracologie concernant les groupes qui devraient faire l'objet d'un dépistage de l'infection tuberculeuse latente

\begin{tabular}{|c|c|}
\hline Groupes à risque & $\begin{array}{l}\text { Groupes qui devraient faire l'objet du dépistage (intervalle d'âge/limite pour le } \\
\text { dépistage) }\end{array}$ \\
\hline $\begin{array}{l}\text { 1. Contacts étroits avec un cas de tuberculose pulmonaire } \\
\text { active } \\
\text { (généralement, les autorités locales de santé publique } \\
\text { participent dans le cadre des enquêtes locales) }\end{array}$ & $\begin{array}{l}\text { Dès que possible après le diagnostic chez le cas index (tous âges) } \\
\text { Contacts regroupés ainsi (11): } \\
\text { - } \quad \text { Priorité élevée : contacts familiaux en plus des contacts non familiaux étroits vulnérables sur le plan immunitaire, tels } \\
\text { - les enfants de moins de } 5 \text { ans } \\
\text { Priorité moyenne : contacts non familiaux étroits exposés au cas chaque jour ou presque, notamment à l'école ou au } \\
\text { - travail } \\
\text { Cas index comme suit (11): } \\
\text { - Tuberculose à frottis positif, cavitaire ou laryngée : le suivi initial des contacts porte sur les contacts de priorité élevée } \\
\text { et ceux de priorité moyenne } \\
\text { Tuberculose pulmonaire non cavitaire à frottis négatif : le suivi initial des contacts concerne les contacts de priorité } \\
\text { élevée seulement }\end{array}$ \\
\hline $\begin{array}{l}\text { 2. Immigrants en provenance d'un pays à forte incidence } \\
\text { de tuberculose }{ }^{1} \\
\text { (soit une incidence } \geq 30 \text { cas } / 100000 \text { personnes pour } \\
\text { toutes les formes de tuberculose active) }\end{array}$ & $\begin{array}{l}\text { Lésions fibronodulaires à la radiographie pulmonaire (habituellement dans le contexte de la surveillance après l'arrivée) } \\
\text { [tous âges] } \\
\text { Tous les enfants et adolescents dès que possible après l'arrivée (jusqu'à l'âge de } 20 \text { ans) } \\
\text { Réfugiés (20-50 ans) } \\
\text { Immigrants et réfugiés atteints d'une autre maladie associée au risque suivant de réactivation de l'infection tuberculeuse } \\
\text { latente }{ }^{2} \text { : } \\
\text { - Risque élevé (tous âges) } \\
\text { - } \quad \text { Risque modéré (jusqu'à l'âge de } 65 \text { ans) } \\
\text { - Risque légèrement accru (jusqu'à l'âge de } 50 \text { ans) }\end{array}$ \\
\hline 3. Autochtones ${ }^{3}$ & $\begin{array}{l}\text { Varie en fonction du risque de tuberculose dans la communauté (12) } \\
\text { Suivi des contacts étroits avec un cas de tuberculose pulmonaire active (comme ci-dessus) [tous âges] } \\
\text { En présence d'une autre maladie associée au risque suivant de réactivation de l'infection tuberculeuse latente² : } \\
\begin{array}{l}\text { - } \\
\text { - }\end{array} \quad \text { Risque élevé (tous âges) } \\
\text { - } \quad \text { Risque légèrement accru (jusqu'à l'âge de } 50 \text { ans) }\end{array}$ \\
\hline 4. Utilisateurs de drogues par injection OU sans-abri & $\begin{array}{l}\text { En présence d'une autre maladie associée au risque suivant de réactivation de l'infection tuberculeuse latente }:^{2} \\
\text { - } \quad \text { Risque élevé }{ }^{2} \text { (tous âges) } \\
\text { - } \quad \text { Risque modéré (jusqu'à l'âge de } 65 \text { ans) } \\
\text { - } \quad \text { Risque légèrement accru (jusqu'à l'âge de } 50 \text { ans) }\end{array}$ \\
\hline 5. Maladies concomitantes (y compris le VIH) & $\begin{array}{l}\text { Le dépistage devrait être envisagé pour toutes les personnes, qu'elles aient déjà été exposées ou non à la tuberculose, } \\
\text { atteintes de certaines autres maladies qui augmentent le risque de réactivation de l'infection tuberculeuse latente }{ }^{2} \text { : } \\
\text { - } \quad \text { Risque élevé (tous âges) } \\
\text { - } \quad \text { Risque modéré (jusqu'à l'âge de } 65 \text { ans) } \\
\text { - } \quad \text { Risque légèrement accru (jusqu'à l'âge de } 50 \text { ans) }\end{array}$ \\
\hline $\begin{array}{l}\text { 6. Voyageurs qui se rendent dans un pays à forte incidence } \\
\text { de tuberculose }{ }^{5}\end{array}$ & $\begin{array}{l}\text { Voyage de } \geq 1 \text { mois comportant un risque très élevé de contact, particulièrement de contact direct avec des patients dans } \\
\text { un hôpital ou un autre milieu intérieur; pourrait comprendre le travail dans une prison, un refuge pour sans-abri, un camp } \\
\text { de réfugiés ou un bidonville (jusqu'à l'âge de } 50 \text { ans en cas de test cutané à la tuberculine unique après le voyage) } \\
\text { Voyage de } \geq 3 \text { mois dans un pays où l'incidence de la tuberculose est }>400 / 100000 \text { habitants }^{1} \text { (tous âges en cas de virage } \\
\text { documenté du test cutané à la tuberculine) } \\
\text { Voyage de } \geq 6 \text { mois dans un pays où l'incidence de la tuberculose est de 200-399/100 } 000 \text { habitants' (tous âges en cas de }^{1} \text { virage documenté du test cutané à la tuberculine) } \\
\text { Voyage de } \geq 12 \text { mois dans un pays où l'incidence de la tuberculose est 100-199/100 } 000 \text { habitants }^{1} \text { (tous âges en cas de } \\
\text { virage documenté du test cutané à la tuberculine) }\end{array}$ \\
\hline 7. Résidents des établissements de soins de longue durée & $\begin{array}{l}\text { Radiographie pulmonaire postéro-antérieure et de profil de base à l'admission pour les personnes appartenant à l'un des } \\
\text { groupes de population à risques }{ }^{6}(>65 \mathrm{ans})(13) \\
\text { Test cutané à la tuberculine de base en deux étapes à l'admission pour les populations à risque }{ }^{6}(\leq 65 \text { ans) (13) } \\
\text { Test cutané à la tuberculine annuel non nécessaire (13) } \\
\text { Le test cutané à la tuberculine n'est plus recommandé comme outil d'évaluation primaire pour le suivi des contacts parmi } \\
\text { les personnes âgées vivant dans un établissement de soins de longue durée; l'accent devrait être mis sur la détection pré- } \\
\text { coce des cas secondaires (13) }\end{array}$ \\
\hline \multicolumn{2}{|c|}{ 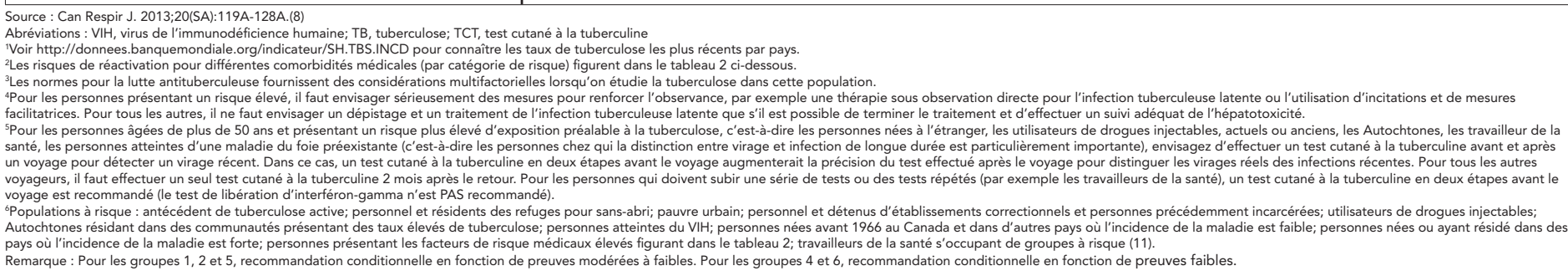 } \\
\hline
\end{tabular}




\section{Progrès des traitements contre l'infection tuberculeuse latente}

Des progrès considérables ont été réalisés dans le traitement à long terme des infections tuberculeuses latentes. Un essai contrôlé de non-infériorité, randomisé, multinational et multicentrique, concernant environ 4000 patients par groupe, a montré que la rifapentine et l'isoniazide (généralement désignés par l'abréviation 3HP) administrés une fois par semaine pour un total de 12 doses en thérapie sous observation directe étaient aussi efficaces que la norme internationale actuelle de 9 mois (252 doses) d'isoniazide autoadministré quotidiennement pour le traitement des infections tuberculeuses latentes (15). Le schéma 3HP a également permis d'obtenir de meilleurs taux d'achèvement du traitement. Un essai contrôlé randomisé (ECR) plus récent réalisé sur une population pédiatrique a également permis d'évaluer l'efficacité du traitement 3HP. Dans cette étude qui concernait 905 patients âgés de 2 à 17 ans, il a été démontré que le schéma 3HP était aussi efficace que l'isoniazide seule pour prévenir l'apparition de la tuberculose chez les enfants. De plus, les taux d'achèvement du traitement étaient plus élevés que pour le traitement standard de 9 mois d'isoniazide (16).

Actuellement, le traitement 3HP contre l'infection tuberculeuse latente n'est disponible au Canada que dans le cadre du Programme d'accès spécial de Santé Canada.

\section{Diagnostiquer rapidement la tuberculose active}

Bien que le taux de transmission de la tuberculose soit faible dans la population générale au Canada, on observe des cas de tuberculose active et de transmission dans les groupes à risque, notamment pendant les éclosions dans les communautés autochtones et dans certains lieux où des personnes sont regroupées (par exemple les refuges, les prisons, les écoles, etc.). Il est essentiel de diagnostiquer rapidement la maladie pour interrompre sa transmission. II s'agit d'une priorité absolue pour le contrôle de la tuberculose.

Pour diagnostiquer la tuberculose, il est indispensable de connaître les antécédents du patient, y compris les principaux facteurs de risque, signes et symptômes, et de disposer de sa radiographie pulmonaire. Pour les nouveaux Canadiens, il est capital de connaître leur pays d'origine et la date de leur arrivée dans le pays. Les données récentes d'une grande étude épidémiologique rétrospective menée en Ontario montrent que les immigrants de 6 pays (Afghanistan, Chine, Inde, Pakistan, Philippines et Vietnam) représentaient $87 \%$ des cas de tuberculose active détectés par le dépistage préalable à l'immigration, tandis que les personnes venant de 10 pays où le taux d'incidence de la maladie est élevé représentaient $80 \%$ des cas de tuberculose active détectés grâce à la surveillance effectuée après l'immigration (7). En outre, cette étude indiquait les catégories d'immigrants associées à un risque plus élevé de tuberculose active après leur arrivée au Canada, les personnes soignantes vivant au domicile des malades et les réfugiés étant les groupes les plus à risque (7). Le risque de réactivation de la tuberculose active est plus élevé dans les deux premières années suivant l'arrivée des immigrants et diminue ensuite chaque année (17). De plus, dans les antécédents médicaux, il est important de déterminer la région de résidence et l'ethnicité des
Tableau 2 : Facteurs de risque de l'évolution vers la tuberculose active chez les sujets présumés être infectés par Mycobacterium tuberculosis

\begin{tabular}{|c|c|c|}
\hline $\begin{array}{l}\text { Le niveau de } \\
\text { risque }\end{array}$ & Facteur de risque & $\begin{array}{l}\text { Risque estimé de } \\
\text { tuberculose }^{1}\end{array}$ \\
\hline \multirow[t]{8}{*}{ FORT } & SIDA & $10-170$ \\
\hline & VIH & $50-110$ \\
\hline & $\begin{array}{l}\text { Greffe d'organe } \\
\text { associée à un traitement } \\
\text { immunosuppresseur }\end{array}$ & $20-74$ \\
\hline & Silicose & 30 \\
\hline & $\begin{array}{l}\text { Insuffisance rénale chronique } \\
\text { nécessitant une hémodialyse }\end{array}$ & $7-50$ \\
\hline & $\begin{array}{l}\text { Carcinome de la tête et du } \\
\text { cou }\end{array}$ & 11,6 \\
\hline & $\begin{array}{l}\text { Infection tuberculeuse } \\
\text { récente ( } \leq 2 \text { ans) }\end{array}$ & 15,0 \\
\hline & $\begin{array}{l}\text { Radiographie pulmonaire } \\
\text { anormale - maladie } \\
\text { fibronodulaire }\end{array}$ & $6-19$ \\
\hline \multirow[t]{4}{*}{ MODÉRÉ } & $\begin{array}{l}\text { Inhibiteurs du facteur de } \\
\text { nécrose tumorale alpha }\end{array}$ & $1,5-5,8$ \\
\hline & \begin{tabular}{|l} 
Diabète sucré \\
(tous les types)
\end{tabular} & $2-3,6$ \\
\hline & $\begin{array}{l}\text { Traitement par des } \\
\text { glucocorticoïdes } \\
(\geq 15 \mathrm{mg} / \text { jour de prednisone })\end{array}$ & 4,9 \\
\hline & $\begin{array}{l}\text { Jeune âge au début de } \\
\text { l'infection ( } 0-4 \text { ans) }\end{array}$ & $2,2-5$ \\
\hline \multirow[t]{4}{*}{$\begin{array}{l}\text { LÉGÈREMENT } \\
\text { ACCRU }\end{array}$} & $\begin{array}{l}\text { Consommation } \\
\text { excessive d'alcool } \\
\text { ( } \geq 3 \text { consommations/jour) }\end{array}$ & $3-4$ \\
\hline & $\begin{array}{l}\text { Insuffisance pondérale } \\
\text { (<90\% du poids idéal; pour } \\
\text { la plupart des personnes, il } \\
\text { s'agit d'un indice de masse } \\
\text { corporelle } \leq 20 \mathrm{~kg} / \mathrm{m} 2 \text { ) }\end{array}$ & $2-3$ \\
\hline & Fumeur (1 paquet/jour) & $1,8-3,5$ \\
\hline & $\begin{array}{l}\text { Radiographie pulmonaire } \\
\text { anormale - granulome }\end{array}$ & 2 \\
\hline
\end{tabular}

personnes nées au Canada, car certaines régions et populations canadiennes, y compris les Autochtones nés au Canada, présentent une incidence plus élevée de tuberculose. Les autres facteurs de risque importants figurent dans le tableau 2.

La présence d'un ou de plusieurs facteurs de risque élevé ou modéré chez un patient présentant des symptômes et un risque épidémiologique de tuberculose peut augmenter considérablement la probabilité de diagnostic de tuberculose (18). Les signes et symptômes de tuberculose sont notamment la perte de poids, la fièvre, la toux chronique, les sueurs nocturnes et l'hémoptysie. Bien que beaucoup de ces symptômes soient 
courants dans d'autres maladies présentes dans les pays où I'incidence de la tuberculose est faible, ils doivent être utilisés en cas d'antécédents qui augmentent la probabilité de tuberculose avant un test. Dans une évaluation récente, plusieurs conseils cliniques importants sont présentés pour répondre à sept questions cliniques, six concernent les antécédents du patient et un concerne le bilan des analyses/des radiographies pour permettre un diagnostic rapide de la tuberculose pulmonaire (18).

Les radiographies pulmonaires, y compris la présentation classique d'une maladie du lobe supérieur chez l'adulte avec maladie fibronodulaire, cavités ou épanchement pleural, sont souvent assez utiles pour le diagnostic. Toutefois, il est important de noter qu'une tuberculose active peut être présente même si la radiographie pulmonaire est normale. Il est essentiel que toutes les personnes chez qui l'on suspecte une tuberculose active subissent un frottis et une culture des expectorations pour confirmer le diagnostic et obtenir la réceptivité. Si une tuberculose active est diagnostiquée, les praticiens de première ligne doivent en informer les services locaux de santé publique et collaborer avec eux. Les professionnels locaux de la santé publique jouent un rôle essentiel en participant à la prise en charge des cas de tuberculose. Ils réalisent notamment les enquêtes sur les contacts fournissent les médicaments contre la tuberculose et administrent les thérapies sous observation directe pour les cas de tuberculose active. De plus, les fournisseurs de soins primaires doivent envisager de référer les patients, notamment les cas complexes, vers des cliniciens de la tuberculose ou des cliniques spécialisées qui proposent des consultations et une assistance à la prise en charge continue.

\section{Conclusion}

Bien que l'incidence de la tuberculose soit faible, les taux d'incidence de tuberculose active dans la population générale n'ont pas évolué de manière significative au cours des dix dernières années, et il existe toujours des disparités importantes dans certaines populations à risque. Dans les efforts réalisés pour éliminer la tuberculose, les cliniciens en soins primaires peuvent faire partie de la solution en mettant l'accent sur 1) le dépistage ciblé et le traitement des infections tuberculeuses latentes et 2) l'identification en temps opportun de la tuberculose active et la référence de ces cas. Les fournisseurs de soins primaires ont une pratique très large et s'occupent inévitablement de populations présentant un risque accru de tuberculose et d'infection tuberculeuse. Ainsi, leur rôle est essentiel pour atteindre les objectifs globaux d'élimination de la tuberculose dans le pays. Ils doivent donc demeurer informés des récentes approches en matière de prévention et de contrôle de la tuberculose, et doivent tenir compte des conseils cliniques et des ressources figurant dans cet article dans le cadre de leur pratique quotidienne, lorsqu'ils prodiguent des soins aux patients à risque.

\section{Conflit d'intérêts}

Aucun.

\section{Références}

1. World Health Organization. Towards tuberculosis elimination: an action framework for low-incidence countries. Geneva (CH): WHO; 2014. http://apps.who.int/iris/bitstre am/10665/132231/1/9789241507707_eng.pdf?ua=1.

2. Stop TB Partnership. The Global Plan to Stop TB 2006-2015. Geneva (CH): World Health Organization; 2006. http://www. stoptb.org/assets/documents/global/plan/globalplanfinal. pdf

3. Public Health Agency of Canada. Tuberculosis prevention and control in Canada: a federal framework for action. Ottawa (ON): The Agency; 2014. http://www.phac-aspc. gc.ca/tbpc-latb/pubs/tpc-pct/assets/pdf/tpc-pcta-eng.pdf.

4. Public Health Agency of Canada. Tuberculosis in Canada 2013 - Pre-release. Ottawa (ON): The Agency; 2015.

5. Long R. Physician experience, public health and the management of tuberculosis. CMAJ. 2006;175(7):759.

6. Centers for Disease Control and Prevention (CDC). Advisory Council for the Elimination of Tuberculosis (ACET) Meeting; 2015 Dec 15-16; Atlanta, GA. http://www.cdc.gov/maso/ facm/facmACET.htm.

7. Khan K, Hirji MM, Miniota J, Hu W, Wang J, Gardam M, et al. Domestic impact of tuberculosis screening among new immigrants to Ontario, Canada. CMAJ. 2015;187(16):E47381.

8. Greenway C, Khan K, Schwartzman K. Tuberculosis surveillance and screening in selected high-risk populations. In: Menzies D, ed. The Canadian tuberculosis standards, 7th Edition. Can Respir J. 2013; 20(Suppl A):119A-28A. http:// tbevidence.org/wp-content/uploads/2013/07/Canadian-TBStandards-7th-Ed-2013.pdf.

9. Immigration, Refugees and Citizenship Canada (IRCC). Panel members' handbook 2013. Ottawa(ON): IRCC; 2015. (Disponible en français : http://www.cic.gc.ca/francais/ ressources/publications/md-manuel/index.asp).

10. Menzies D, Alvarez GG, Khan K. Treatment of latent tuberculosis infection. In: Menzies D, ed. The Canadian tuberculosis standards, 7th edition. Can Respir J. 2013;20(Suppl A):44A-53A. http://tbevidence.org/wpcontent/uploads/2013/07/Canadian-TB-Standards-7thEd-2013.pdf.

11. Rea E, Rivest P. Contact follow-up and outbreak management in tuberculosis control. In: Menzies D, ed. The Canadian tuberculosis standards, 7th Edition. Can Respir J. 2013;20(Suppl A):108A-18A. http://tbevidence.org/ wp-content/uploads/2013/07/Canadian-TB-Standards-7thEd-2013.pdf.

12. Alvarez GG, Orr P, Wobeser WL, Cook V, Long R. Tuberculosis prevention and care in First Nations, Inuit and Métis people. In: Menzies D, ed. The Canadian tuberculosis standards 7th Edition. Can Respir J. 2013;20(Suppl A):129A35A. http://tbevidence.org/wp-content/uploads/2013/07/ Canadian-TB-Standards-7th-Ed-2013.pdf. 
13. Ogunremi T, Menzies D, Embil J. Prevention and control of tuberculosis transmission in health care and other settings. In: Menzies D, ed. The Canadian tuberculosis standards, 7th Edition. Can Respir J 2013;20(Suppl A):136A-51A. http:// tbevidence.org/wp-content/uploads/2013/07/Canadian-TBStandards-7th-Ed-2013.pdf.

14. McGill University. The Online TST/IGRA Interpreter. Version 3.0. http://www.tstin3d.com/.

15. Sterling TR, Villarino ME, Borisov AS, Shang N, Gordin F, Bliven-Sizemore $E$, et al. Three months of rifapentine and isoniazid for latent tuberculosis infection. $\mathrm{N}$ Engl J Med. 2011;365:2155-66.
16. Villarino ME, Scott NA, Weis SE, Weiner M, Conde MB, Jones $B$, et al. Treatment for preventing tuberculosis in children and adolescents: a randomized clinical trial of a 3-month, 12-dose regimen of a combination of rifapentine and isoniazid. JAMA Pediatr. 2015;169(3):247-55.

17. Cain KP, Benoit SR, Winston CA, Mac Kenzie WR. Tuberculosis among foreign-born persons in the United States. JAMA. 2008;300(4):405-12.

18. Long R. Making a timely diagnosis of tuberculosis. Can Respir J. 2015;22(6):317-21. 